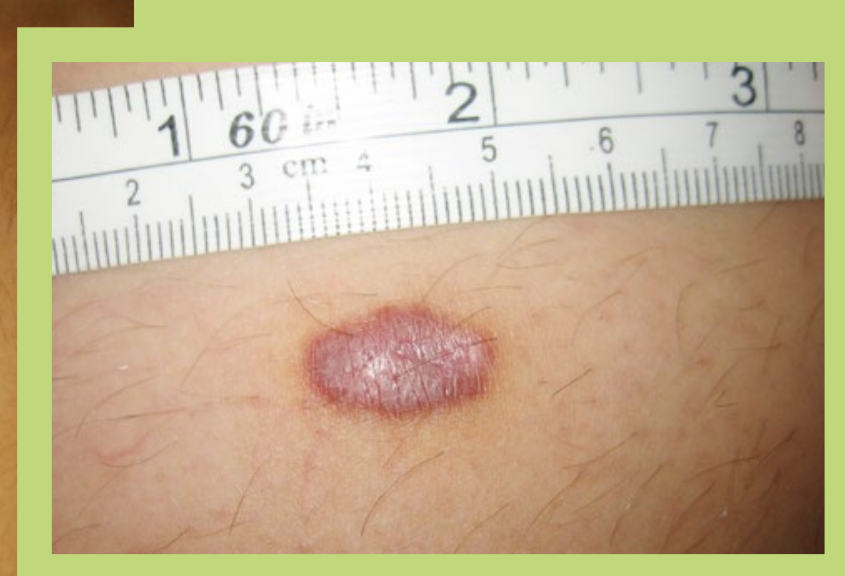

Rot-braune, schmerzlose, nicht juckende Hautläsionen am Stamm.

\title{
Hautbefund
}

\section{Kutane Warnlampe für den HIV-Test}

Ein 32-jähriger, $172 \mathrm{~cm}$ großer und $65 \mathrm{~kg}$ schwerer Akademiker stellte sich im Sommer 2016 zunächst bei seinem Hausarzt vor. Der Patient hatte bemerkt, dass am Stamm und an den proximalen Extremitäten kleine, rot-blaue Flecken aufgetreten waren, die nicht juckten und nicht schmerzten. Außerdem war es zu einer Gewichtsabnahme um ca. $5 \mathrm{~kg}$ und $\mathrm{zu}$ einem trockenen Husten gekommen. Mit der Verdachtsdiagnose eines Kaposi-Sarkoms überwies der Hausarzt den Patienten in unsere Spezialambulanz.

Wegen der Hautveränderungen und der anamnestischen Angabe sexueller Kontakte mit Männern führten wir einen HIV-Test durch, der sich als positiv erwies. Tatsächlich ist der Hautbefund klassisch für ein beginnendes kutanes Kaposi-Sarkom. Untermauert wurde dies durch einen positiven Test auf das humane Herpesvirus 8, das pathogenetisch ursächlich für diese Art von Spindelzellproliferation ist.

Bei der körperlichen Untersuchung zeigte sich ein Puls von 92 pro Minute, eine Atemfrequenz von 20/min in Ruhe, ein Blutdruck von 110/70 mmHg und eine Körpertemperatur von $37,0^{\circ}$. Die Auskultation und Perkussion der Lunge ergab normale Befunde. Beim Blick in den Mund fiel zusätzlich ein
Mundsoor auf. Mukosale Kaposi-Herde waren nicht zu erkennen. Weitere Untersuchungen ergaben pulmonale Verdichtungsherde und mediastinale Lymphknotenvergrößerungen, die wir als konsistente Hinweise auf eine viszerale Beteiligung werteten.

Bei einer CD4-Zellzahl von 13/ $\mu$ l (normal > 400) und einer HI-Virusmenge von $>300.000 \mathrm{Kopien} / \mathrm{ml}$ leiteten wir eine antiretrovirale Kombinationstherapie und eine Cotrim-Prophylaxe ein. Im weiteren Verlauf erreichte der Patient rasch das Therapieziel einer Viruslast von $<50$ Kopien/ml. Auch die Kaposi-Herde wurden allmählich kleiner.

Das Kaposi-Sarkom ist eine seltener gewordene, aber immer noch aktuelle „Warnlampe“, die zum HIV-Test führen muss. Nicht bei jedem Patienten ist wie bei diesem Patienten die alleinige Immunrekonstitution ausreichend, das Sarkom zurückzudrängen. In seltenen Ausnahmen kann eine Chemotherapie mit liposomalem Doxorubicin notwendig werden.

\footnotetext{
$\rightarrow$ Prof. Dr. med. Johannes R. Bogner

Medizinische Klinik und Poliklinik IV, Klinikum der Universität

München, Pettenkoferstr. 8a, D-80336 München
} 